University of Nebraska - Lincoln

DigitalCommons@University of Nebraska - Lincoln

Faculty Publications from the Department of Engineering Mechanics

$10-2006$

\title{
Role of Atomic Scale Interfaces in the Compressive Behavior of Carbon Nanotubes in Composites
}

S. Namilae

FAMU-FSU College of Engineering, Florida State University, 2525 Pottsdammer Road, Tallahassee, FL

Namas Chandra

University of Nebraska - Lincoln, nchandra2@unl.edu

Follow this and additional works at: https://digitalcommons.unl.edu/engineeringmechanicsfacpub

Part of the Mechanical Engineering Commons

Namilae, S. and Chandra, Namas, "Role of Atomic Scale Interfaces in the Compressive Behavior of Carbon Nanotubes in Composites" (2006). Faculty Publications from the Department of Engineering Mechanics. 51.

https://digitalcommons.unl.edu/engineeringmechanicsfacpub/51

This Article is brought to you for free and open access by the Mechanical \& Materials Engineering, Department of at DigitalCommons@University of Nebraska - Lincoln. It has been accepted for inclusion in Faculty Publications from the Department of Engineering Mechanics by an authorized administrator of DigitalCommons@University of Nebraska - Lincoln. 


\title{
Role of Atomic Scale Interfaces in the Compressive Behavior of Carbon Nanotubes in Composites
}

\author{
S. Namilae and N. Chandra \\ Department of Mechanical Engineering, FAMU-FSU College of Engineering, \\ Florida State University, 2525 Pottsdammer Road, Tallahassee, FL 32310, USA
}

Corresponding author - N. Chandra

\begin{abstract}
Carbon nanotubes (CNT) are potentially promising fibers for ultra high strength composites. In order to fully harness the outstanding mechanical properties of carbon nanotubes as fiber reinforcements, it is essential to understand the nature of load transfer between fiber and matrix under various types of loading conditions that include tension, compression, torsion and a combination thereof. In this paper, we study the compressive behavior (buckling and post-buckling) of carbon nanotubes in the neat form, when they are embedded in polyethylene matrix and with interface chemical modifications using molecular dynamics simulations based on Tersoff-Brenner potential. It is observed that the critical load for buckling increases only very marginally for nanotubes embedded in polythene matrix (with non-bonded interface) compared to neat CNTs. When CNTs are chemically bonded to the matrix, the compressive behavior occurs in two phases; pre- and post-buckling phases. First, the critical stress for buckling is found to reduce because the change in chemical bonding induces deviation from perfect cylindrical structure. In the post-buckling phase, however, the nanotubes behave similar to short fibers and deform by crushing. The results are compared with continuum solutions, wherever applicable. It is shown that the continuum solutions should be applied carefully whenever the effect of nanoscale interfaces becomes a factor.
\end{abstract}

Keywords: carbon nanotubes, interfaces, buckling, molecular dynamics

\section{Introduction}

The combination of high strength, stiffness and aspect ratio makes carbon nanotubes ideal reinforcements for ultra high strength composites. Stiffness of CNTs measured experimentally [1] and calculated from simulations [2] is of the order of $1000 \mathrm{GPa}$, while the nearest competitive fiber (SiC whiskers) has utmost $400 \mathrm{GPa}$ in stiffness [3]. CNTs have tensile strength of up to $150 \mathrm{GPa}$ [4]. The range of elastic deformation and fracture strain are also extremely high $[5,6]$. This combination of mechanical properties has raised the possibility of obtaining super-strong and stiff composites with CNTs as reinforcements. Further, the excellent electrical and optical properties of CNTs facilitate development of multifunctional products [7]. Numerous research- ers are fabricating composites in bulk and thin film forms with different matrix materials.

The typical length of CNTs is of the order of a few microns while the diameters range from less than a nanometer (for single wall nanotubes) to about $30 \mathrm{~nm}$ (for multiwall nanotubes) $[8,9]$ resulting in a large aspect (length to diameter) ratio. Because of this large aspect ratio and tubular structure, buckling and bending are easily possible for CNTs per se and in composites. In addition, carbon nanotubes have been reported to be significantly resilient and recover elastically from heavily buckled states [10, 11]. Future applications of nanotubes can possibly rely on their high strength properties as well as their ability to absorb large amounts of strain energy.

Buckling and compressive deformation of nanotubes 
have been the subject of numerous experimental and computational studies. Experimental investigators have observed micrographs of nanotubes in buckled state both as individual nanotubes $[10,12,13]$ and when embedded in composite [14, 15]. Computational studies thus far have been based on both atomistic techniques such as molecular dynamics, and continuum-based shell and beam theories. Yakobson and coworkers [11] obtain a good match between molecular dynamics calculations and continuum shell theory-based calculations for small aspect ratios. Harik [16] has investigated the ranges of applicability of continuum models by comparing with molecular dynamics simulations. Ru $[17,18]$ has derived analytical expressions for ropes of nanotubes and multiwall nanotube with shells interacting through Van Der Wall forces. Li and Chou [19] use structural mechanics model to compare the buckling force in axial compression and bending modes. Garg and Sinnott [20] have studied the compressive behavior of functionalized CNTs. There have been several other investigations on buckling of nanotubes using continuum beam or shell models [21, 22, 23]. Though this large volume of work concentrates on buckling of nanotubes by themselves, there have been relatively few studies on the compressive behavior of nanotubes when embedded in composites $[15,18]$.

An important issue while studying the buckling behavior of CNT composite is the nature of interface and the extent to which load transfer occurs between the fiber and the matrix. Because of the nanoscale dimensions of CNT fibers, the interfacial region surrounding the fiber is also in nanometer dimensions. Thus, the study of nanotube buckling raises some very interesting mechanics issues not found in continuum scale problems. Buckling is influenced by the forces exerted in the interfacial region as well as the elastic properties of surrounding matrix. In the case of carbon nanotubes, there are conflicting reports regarding the strength of interfaces. Several researchers report evidence for load transfer based on microstructural and spectroscopic observations [9, 23-25]. Ding and coworkers [25] have observed polymer sheathing of nanotubes in PMMAnanotube composite indicating strong interaction between nanotubes and PMMA. Some investigators report low interfacial load transfer $[14,26]$. In addition, the measured values of stiffness of CNT-based composites reported by various researchers are much lower than possible theoretical values. For example, Andrews and coworkers [27] report normalized Young's modulus (stiffness of composite/ stiffness of matrix) values in the range of 1.2-2.5 for polystyrene matrix reinforced with $2.5-25 \mathrm{vol} \% \mathrm{CNT}$. The comparable theoretical maxima and minima based on parallel (iso-strain) and series (iso-stress) models are in the range of 14-140 and 1.03-1.33, respectively. One of the possible reasons for low stiffness, assuming that the orientation is totally random, is inadequate interfacial strength. Chemical modification of the surface of nanotube is expected to enhance the interface shear strength. Researchers have successfully modified the surface of CNTs with different functional groups [28-30] and the use of these fibers for composites is expected to enhance the mechanical properties [31,32]. In an earlier study we have used molecu- lar dynamics simulations of pullout tests to show that the chemical modification of nanotube surfaces through functionalization is expected to enhance the interface strength significantly [32].

The objective of the present paper is to understand the role of atomic scale interfaces in the load transfer characteristics of nanotubes vis-à-vis compressive behavior of nanotube reinforced composites. We first examine the compressive behavior of both neat CNTs and CNTs embedded in polyethylene matrix with only van der Waals interaction between the matrix and fiber. Next, we study the effect of chemically bonded CNT-matrix interfaces on both buckling and post-buckling behaviors. Additionally, the numerical simulation results are compared with continuum-based solutions to elucidate the difference between conventional and atomic scale interfaces.

\section{Buckling of neat carbon nanotubes}

We primarily employ molecular dynamics simulations in the present study. It is very critical to choose appropriate potential that has capability to model the complex chemical bonding behavior accurately under dynamical loading conditions. Tersoff-Brenner bond-order potential [33, 34] has coordination dependant terms, which enable modeling bond conjugations in carbon, and has been used by numerous investigators to study processes such as deformation of carbon nanotubes [2, 5, 11, 20,35], and various surface chemical reactions in carbon-hydrogen systems [33, 36]. We use Tersoff-Brenner potential for all the simulations on $(10,10)$ CNTs of different lengths at temperature of $300 \mathrm{~K}$. Compression of CNTs (with and without polymer matrix) is simulated by prescribing end displacement $(0.05 \AA)$ at both the ends followed by equilibration (for 1000 steps).

Figure 1 shows the compressive stress-displacement plot for $(10,10)$ nanotubes of different aspect ratios (10-22).

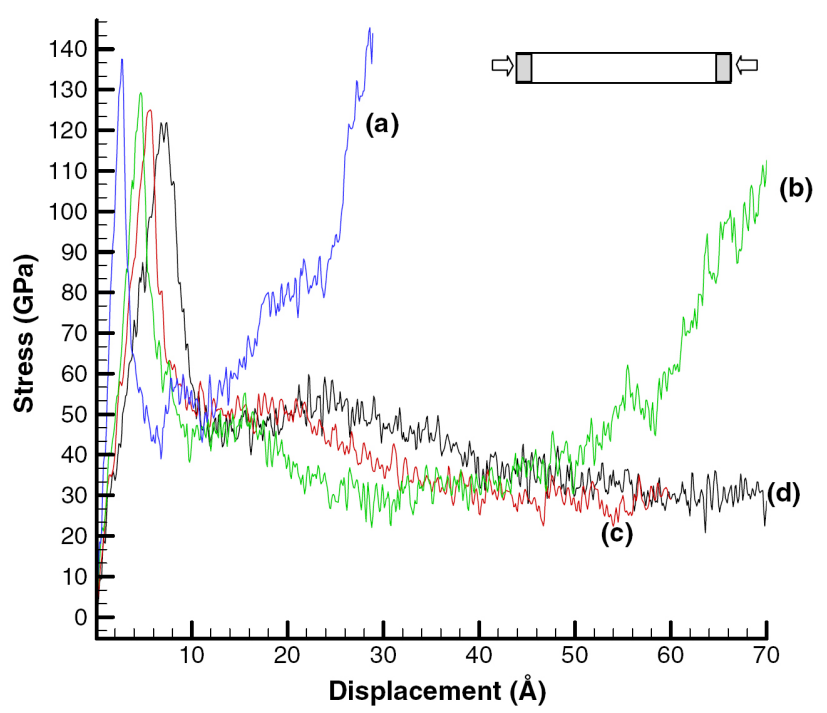

Figure 1. Compressive stress vs. displacement plot for $(10,10)$ CNT of different aspect ratios. Aspect ratio for: (a) 10; (b) 15; (c) 18 ; (d) is 22 . 
Stress is calculated as the average of reaction force experienced by the fixed atoms over 100 time steps, divided by the cross sectional area (with thickness of $3.4 \AA$ ). Though it is customary to study critical loads rather than stress in buckling, we prefer the latter, since we study both preand post-buckling behaviors. However, the concept of force and stress may be interchangeably used in the prebuckling stages, while stresses are the more appropriate measure in post-buckling conditions. It can be observed from the plot that the critical stress (or load) for buckling decreases as the aspect ratio of CNT increases (case ' $a$ ' to case ' $d$ '). This trend of increase in aspect ratio leading to decrease in critical force is similar to that in continuumbased models [39].

In order to understand the pre-buckling behavior, we have plotted the average bond lengths for the same set of simulations in Figure 2. It is evident from the figure that the increase in compressive stress is a direct consequence of decrease in bond length. For example, nanotube with an aspect ratio of 10, corresponding to plot (a) in Figure 1 the average bond length reduces from $1.426 \AA$ initially to $1.396 \AA$ at the peak stress region. Beyond the peak value some regions of the nanotube deviate from the original cylindrical configuration leading to localized out of plane deformation. Such deformation accommodates the imposed compressive displacement, despite the fact that average bond length relaxes from the peak value. This relaxation leads to the decrease in stress as seen in Figure 1.

It is interesting to observe that in case "a" (aspect ratio $=10)$ there is a rapid increase in compressive stress beyond a displacement of $10 \AA$. Similar trend is seen for case " $b$ " and is expected to occur in cases " $c$ " and " $d$ " at much higher displacement values (not shown for practical reasons). The rapid increase in stress occurs, when both the moving ends of CNT are in close proximity of each other. During this phase the central region of CNT is severely distorted and carbon atoms are brought very near to each other. This "crushing process" generally occurs

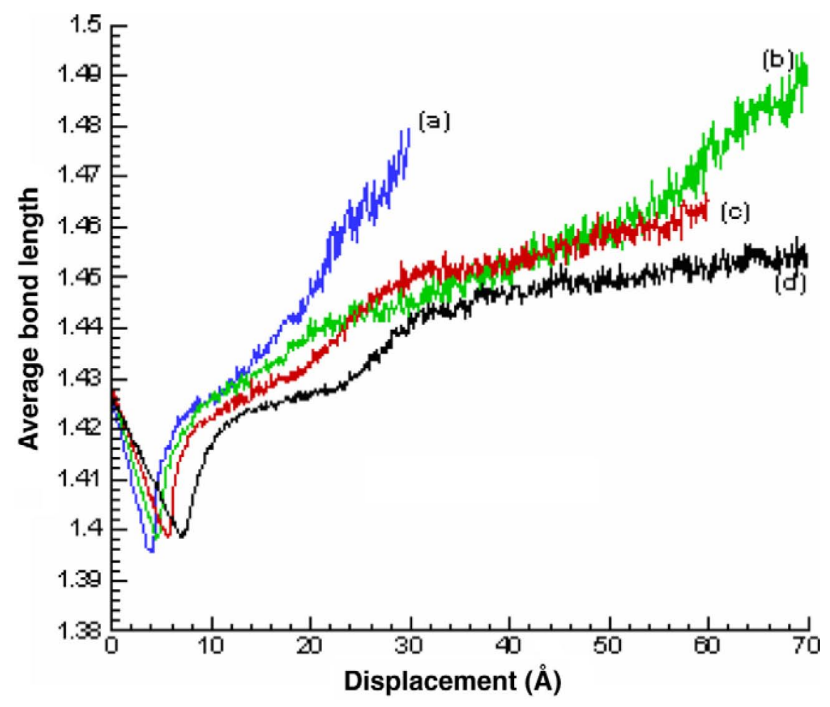

Figure 2. Variation of average bond length with applied displacement. when the two ends are brought to some critical distance, and thus occurs at higher applied displacement for longer nanotubes.

\section{Buckling of carbon nanotube embedded in polythene matrix}

We now proceed to study the buckling of nanotubes embedded in polymer matrix. The polymer consists of 36 polythene chains of length approximately same as that of the embedded CNT and aligned along the length of CNT as shown schematically in Figure 3. The density of polythene in the model is about $0.8 \mathrm{~g} / \mathrm{cc}$, which is comparable to commercial polythene. The non-bonded interactions between the polymer chains and CNT are modeled using 126 Lennard-Jones potential given by Equation (1) with the parameters $\varepsilon=2.86 \mathrm{meV}$ and $\sigma=3.4 \AA$ [37]

$$
V(r)=4 \varepsilon\left(\left(\frac{\sigma}{r}\right)^{12}-\left(\frac{\sigma}{r}\right)^{6}\right)
$$

The C-C and C-H chemical bonds are still modeled using Tersoff-Brenner potential while the Lennard-Jones potential is used for non-bonded interactions only. Compressive deformation is applied on the CNT embedded in polythene as described earlier. Our simulations indicate that the deformation pattern is observed to remain strikingly similar to that of free nanotubes (with no matrix). This shows that the forces exerted by van der Waals interaction with matrix are not large enough to cause changes in CNT morphology during compression. It is interesting to note that Lourie and coworkers [15] have observed that deformation patterns of bent nanotubes embedded in matrix are similar to those of free nanotubes from earlier experiments [13]. This is in agreement with our simulations. Figure 4 shows the compressive stress displacement plots for $(10,10)$ CNT with aspect ratio 15 embedded in crystalline polythene matrix. There is a marginal increase in the

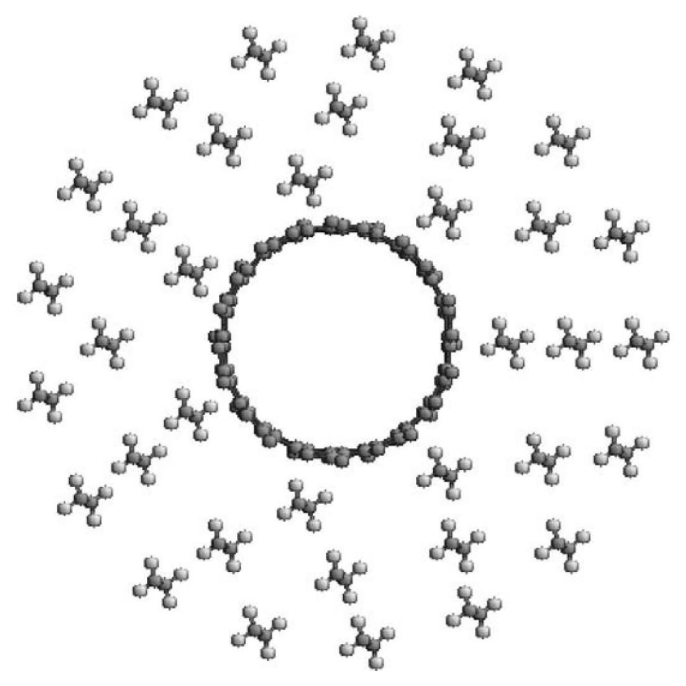

Figure 3. Schematic of CNT embedded in crystalline polyethylene matrix. 


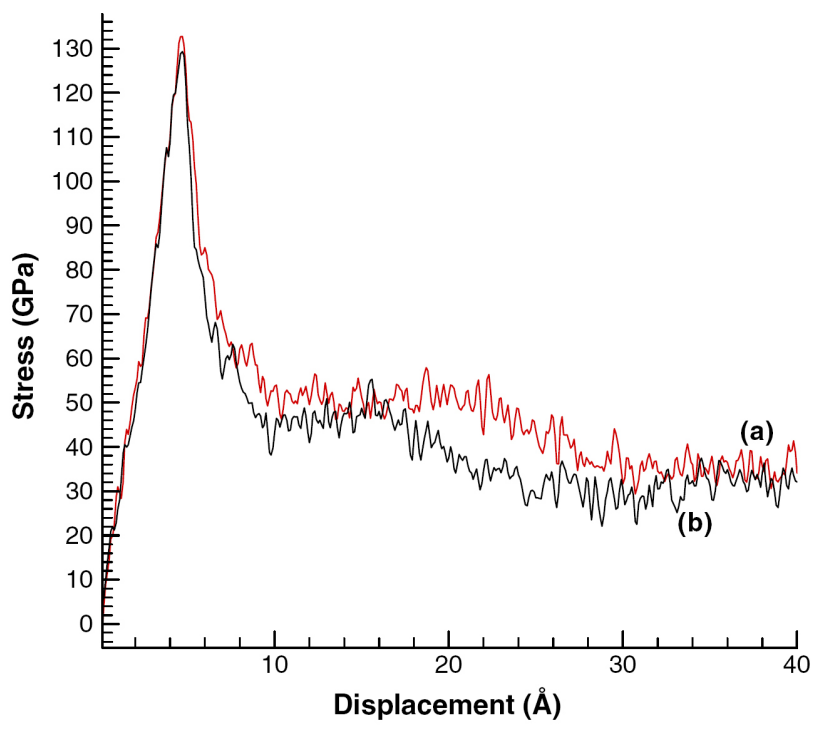

Figure 4. Compressive stress vs. displacement plot for $(10,10)$ CNT embedded in polyethylene composite (plot (a)). Plot (b) is the stress-displacement plot for neat nanotube.

critical stress for the CNT embedded in polythene matrix. In addition, the stress during the post-buckling phase is higher for the composite. Similar trend is observed for fibers of different aspect ratios.

Thus, our simulation shows that the compressive behavior of carbon nanotubes is affected only slightly, whether they are embedded in a composite or not, as long as the interfacial load transfer is governed only by the van der Waals forces. This is contrary to continuum-based intuition that matrix will influence the buckling behavior. In order to explore this issue further, we turn to continuum-based models.

Continuum-based shell and beam models have been applied with reasonable success to CNTs [11]. Winkler model $[38,39]$ has been commonly used to study the buckling of fibers in elastic medium. Louri and coworkers [15] have used this model to estimate the compressive strength of CNTs. Ru [18] has recently combined continuum shell equations with Winkler model for a multiwall nanotubes embedded in elastic medium. This expression can be simplified to obtain the critical stress of a single walled nanotube embedded in an elastic medium as:

$$
\begin{aligned}
\sigma^{\mathrm{cr}}= & \frac{P^{\mathrm{cr}}}{2 \pi R h} \\
= & \frac{D \pi}{2 R h m^{2} L^{2}}\left(m^{2}+\left(\frac{n L}{\pi R}\right)^{2}\right)+\frac{m^{2} L^{2} E}{2 \pi^{3} R^{3}\left(m^{2}+\left(\frac{n L}{\pi R}\right)^{2}\right)} \\
& +\frac{k L^{2}}{2 \pi^{3} m^{2} R h}
\end{aligned}
$$

where $P^{\mathrm{cr}}$ is the critical load, $D$ is the bending stiffness of CNT; $L, R$ and $h$ are the length, radius and thickness of CNT, respectively. Critical stress at the onset of buckling is obtained by minimizing the right-hand side of the above equation with respect to integers $m$ and $n$. Physically they represent the number of waves nanotube subdivides at buckling. $k$ is the Winkler's spring constant or the foundation modulus of the matrix [38,40]. It should be noted that the first two terms of the right-hand side of the above equation correspond to buckling of CNT, while the third term is the increase in load due to the surrounding medium. For $m=1$ and $n=1$ the increase in critical load is

$$
\Delta \sigma^{\mathrm{cr}}=\frac{k L^{2}}{2 \pi^{3} R h}
$$

The foundation modulus $k$ depends on the elastic modulus of matrix, the aspect ratio of nanotube and fiber-matrix interaction. Limiting value of $k$ for a matrix with elastic modulus $E_{\mathrm{m}}$ and Poisson ratio $v$, can be simplified as shown below $[38,40]$

$$
k=4 \pi \frac{E_{\mathrm{m}}(1-v)}{(1+v)(3-4 v)} \frac{1}{K_{0}(\pi R / L)}
$$

Here, the corresponding increase in stress $\Delta \sigma^{\mathrm{cr}}$ is

$$
\Delta \sigma^{\mathrm{cr}}=\frac{2 E_{\mathrm{m}}(1-v) L^{2}}{\pi^{2}(1+v)(3-4 v) R h} \frac{1}{K_{0}(\pi R / L)}
$$

For the geometry of nanotubes considered in the Figure 3, assuming a value of 0.3 for Poisson's ratio, the increase in critical stress must be of the order of $20 E_{\mathrm{m}}$ (about $30 \mathrm{GPa}$, considering the values for polythene). Using molecular dynamics simulations, we observe that there is a much lesser increase in critical stress (about $5 \mathrm{GPa}$ ) compared to that predicted by the above equations.

This discrepancy can be explained when we consider the difference between interfaces at nanoscale and micro scale (for that matter all other larger scales). Interfaces in conventional composites often consist of intermediate phases formed by chemical reaction between matrix and fiber and are typically a few microns thick [41]. When a fiber buckles under compressive load, a small deformation of the fiber is sufficient for the interface (and matrix) to be loaded and hence exert a reaction on the fiber. Now consider the onset of buckling in CNT-polythene composite. The equilibrium spacing between nanotubes in multi-wall nanotubes is about $3.4 \AA$; hence the average initial spacing between the nanotube and polythene chains in the first circular layer of matrix (see Figure 3) was chosen to be $3.4 \AA$. This distance is significant when compared to the radius of $(10,10)$ CNT which is $6.78 \AA$. The force between the fiber and matrix is only due to van der Waals interactions; when the lateral displacement due to buckling is very small, there is essentially no effect on the matrix. The relative motion during buckling is small to cause any substantial changes in the interaction forces given by Equation (1), compared to the energy expended in deforming the matrix which is the basis for the Winkler model. This explains the very small increase in critical stress obtained in the computation. Deformation of matrix due to displacing of fiber occurs only during the postbuckling phase and does not affect the critical stress for 
buckling. The use of continuum model predicts more increase in critical stress than that observed using molecular dynamics simulation; hence the atomistic issues must be carefully analyzed before applying continuum model to this problem.

\section{Compressive behavior of nanotube with chemically bonded interfaces}

Interfaces in nanocomposites should be distinguished from conventional composites wherein the fiber-matrix interface consists of distinctly different phases from that of both fiber and matrix. For example in the case of $\mathrm{SiC}$ reinforced titanium metal matrix composites, interface consists of a series of intermediate chemical phases that span a few micron thickness. Here, we have an "interphase" with its own chemical and mechanical characteristics that affect the overall composite behavior.

Nanoscale interfaces considered currently are of the order of utmost a few atomic layers. Even when the matrix and fiber are chemically bonded, the interface is not continuous and exists only at discrete locations. In such a case, the mechanical behavior of interface is sum total of the effect of these discrete bonds. As an example of this system, we have attached butene $\left(\mathrm{C}_{4} \mathrm{H}_{8}\right)$ molecules to CNTs at selected locations. The interface strength is governed by the number of such attachments (per unit area) which is varied in the present study. Axial displacements are applied as described earlier to produce compressive deformation. While one end of the butane hydrocarbon molecule (acting as interface) is chemically bonded to the $\mathrm{CNT}$, the other end is fixed as a boundary condition. Fixing the matrix end and allowing the motion at the nanotube end enables us to evaluate the interface properties in terms of force vs. differential displacement. This relation yields the parameters needed for the celebrated cohesive zone model of the interface. It has been shown that the strength of interfaces increases significantly by increasing the number of chemical attachments per unit area [32].

Figure 5 shows the compressive stress vs. displacement plot for the interface discussed above. Stress is calculated as the average of reaction force experienced by the fixed atoms over 100 time steps, divided by the cross sectional area, while the displacement is the applied displacement on the fixed ends. Plot (a) in Figure 5 is that of neat $(10,10)$ CNT while plots (e)-(g) correspond to nanotube with 80, 40 and 20 chemical attachments, the matrix end of the chemical attachments being fixed. Plots (b)-(d) are for functionalized nanotubes in which the matrix end of chemical attachments are free and will be discussed later. It can be observed from Figure 5 that the critical stress for buckling is reduced (by $10-15 \%)$ in nanotubes with chemically bonded interfaces. The decrease in critical stress is counter intuitive since the attachment can be presumed to reduce the total length into smaller segments; critical load for buckling is expected to increase for columns of smaller length. For example, if a column (length $L$, radius $r$ ) is subdivided by $n$ uniformly spaced rigid restraints, then:
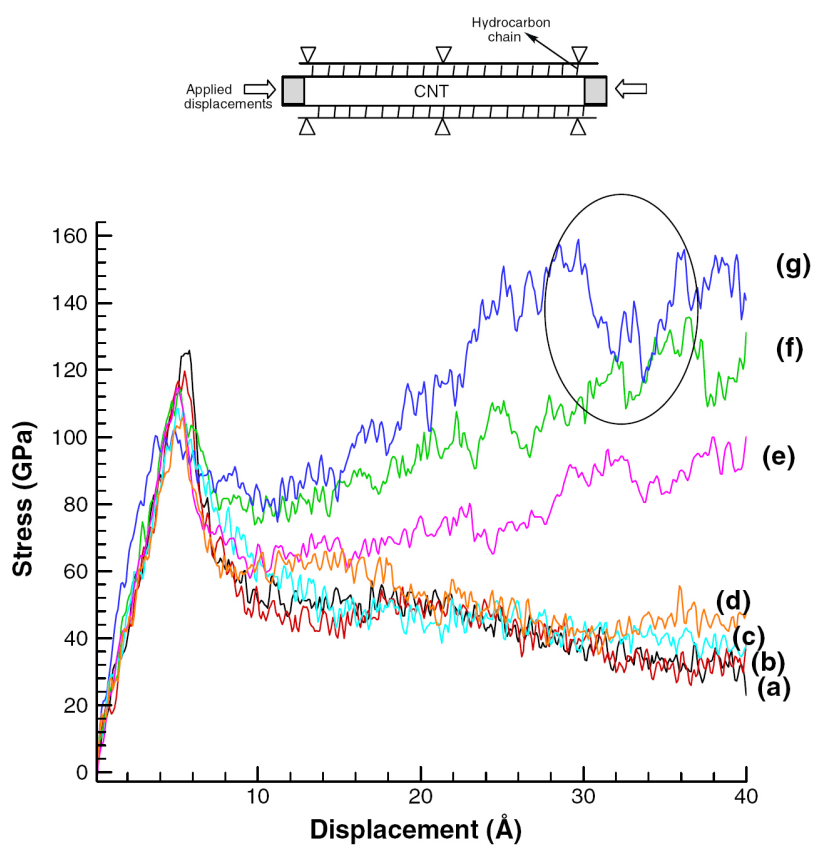

Figure 5. Compressive stress vs. applied displacement for (a) $(10,10)$ CNT; (b-d) functionalized CNT; (e-g) composite with strong interface. Inset shows the boundary conditions schematically.

$$
\sigma_{\mathrm{cr}}=\frac{P_{\mathrm{cr}}}{A}=n^{2} \frac{\pi^{2} E}{(L / r)^{2}}
$$

As $n$ increases, the continuum theory predicts that critical stress will increase by $n^{2}$. However, the atomistic simulations show that the critical stress for buckling reduces.

The decrease in critical stress can be explained by considering the geometrical structure of chemically bonded CNTs. Nanotubes have planar graphitic structure with $\mathrm{sp}^{2}$ hybridization. When the surface of CNT is chemically modified by attaching hydrocarbon molecule, the bond structure changes from $\mathrm{sp}^{2}$ to $\mathrm{sp}^{3}$ resulting in a spike in CNT. Figure 6 shows the radius of curvature of CNTs with and without chemical attachments. The peaks in Figure 6 correspond to the locations of chemical attachment; it can be observed that the radius at these locations is about $7.3 \AA$ as compared to $6.78 \AA$ where there are no attachments. In addition, it can be seen that there is a decrease in radius of curvature in regions adjoining the location of attachment. Both these factors cause a deviation from the ideal cylindrical structure of nanotubes resulting in condition similar to mechanical serrations in the region of chemical attachments.

Buckling is essentially a displacement controlled process, wherein eccentricity arising out of material, geometry, or boundary load causes instability leading to failure. As the length increases, the required eccentricity to cause instability reduces, thus decreasing the critical load. Any additional eccentricity present due to geometry or loading causes instability to occur at even lower loads. For example, consider a column with initial curvature corresponding to a deflection of $v_{0}(x)$; then the total deflection in the 


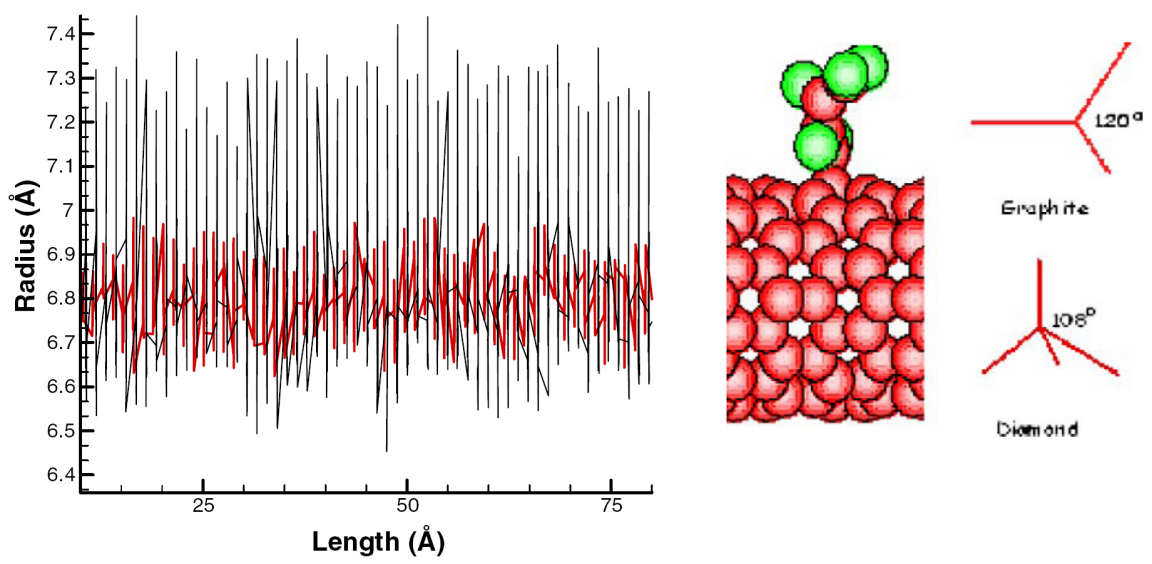

Figure 6. Variation of radius along the length for stabilized CNT with (black line) and without (red line) chemical attachments. The peaks (in black) correspond to the locations of hydrocarbon attachments. Schematic shows $\mathrm{sp}^{3}$ and $\mathrm{sp}^{2}$ hybridization.

column can be obtained as $v_{\text {tot }}=v_{0}(x)+v$. The differential equation for buckling now becomes

$$
\mathrm{EI} \frac{\mathrm{d}^{2} v}{\mathrm{~d} x^{2}}=-P v_{\text {tot }}=-P\left[v_{0}(x)+v\right]
$$

If the initial deflection is sinusoidal with amplitude $a_{0}$ given by $v_{0}(x)=a_{0} \sin (\pi x / l)$, compressive stress experienced by the column is given by

$$
\sigma=\frac{P}{A}\left[1+\frac{a_{0} A}{S} \frac{1}{1-\left(P l^{2} / \mathrm{EI}\right)}\right]
$$

where $S$ is the section modulus and $P$ is the applied load. Equation (8) determines the compressive stress experienced by the column. As this stress is higher for the same external load in the presence of an eccentricity, there is a corresponding decrease in the critical load for buckling. In the case of functionalized CNT due to the hybridization effect noted earlier, the mechanical serrations introduce additional eccentricity. Thus, there are two competing factors, one caused by $n$ restraints (Equation (6)) and the other by initial curvature due to hybridization (Equations (7) and (8)). Equation (6) presumes that the column is held pinned at those $n$ points; however, this does not happen in the case of nanotube with chemical attachments. Even though the matrix end of chemical attachments is held fixed, the chemical bonds near the fiber are flexible and there is no load transfer during the pre buckling stage. The curvature effect then becomes the only effective process, increasing the critical stress for buckling.

In Figure 5, plot (a) corresponds to neat nanotube, plots (b)-(d) represent nanotubes with chemical attachments that are free at matrix end, while (e)-(g) are for similar cases with the matrix end of chemical attachments fixed. No significant difference is observed during pre-buckling and critical stress between the cases when the matrix end is free or held fixed. This is clearly because no load transfer occurs during this stage of loading, as discussed earlier.
We now proceed to discuss the post-buckling phase of nanotube fibers with bonded interfaces. If we compare the plots (b)-(d) and (e)-(g) of Figure 5 it is clear that there is significant effect of interfaces on the post-buckling behavior. It can be observed from the figure that the compressive stress experienced by the nanotube during post-buckling is even more than the critical stress for buckling. In addition there is a clear effect of the density of chemical attachments; the stress experienced by the fiber with more number of attachments (plot $(\mathrm{g})$ ) is significantly higher than that with smaller number of attachments (plot (e)). These effects indicate that unlike pre-buckling stage, there is significant load transfer occurring in the post-buckling deformation.

In order to study the stress distribution in the interior regions of the nanotube we use a local stress measure known as Lutsko stress [42]. The details of different atomic scale stress measures are discussed elsewhere [35, 43]. In its general form Lutsko stress $\sigma_{i j}^{\text {Lutsko }}$ to is given by

$$
\sigma_{i j}^{\text {Lutsko }}=\frac{1}{\Omega^{\text {Avg }}} \sum_{\alpha=1, n}\left(\frac{1}{2} m^{\alpha} v_{i}^{\alpha} v_{j}^{\alpha}+\sum_{\beta=1, n} r_{\alpha \beta}^{j} f_{\alpha \beta}^{i} l_{\alpha \beta}\right)
$$

Here, $\Omega^{\mathrm{Avg}}$ is the averaging volume, $v, r, f$ are velocity force and radial vectors, $\alpha$ and $\beta$ are the atomic indices, $i$ and $j$ are the indices of the stress tensor. $l_{a \beta}$ denotes the fraction of $\alpha-\beta$ bond lying inside the averaging volume. Lutsko stress enables the calculation of local stresses by selecting any averaging volume smaller than the total volume. The large deformation experienced in buckling makes it very difficult to calculate current averaging volume $\left(\Omega^{\mathrm{Avg}}\right)$, and in this analysis we use the initial averaging volume instead.

Plots (a)-(c) in Figure 7 correspond to different regions of nanotube as shown in the inset, with the interfacial region consisting of 80 chemical attachments. Plots (d)-(f) correspond to similar regions for functionalized nanotube without fixing the matrix end. It can be clearly noted from Figure 7 that stress is not transferred to the central region completely either during buckling or post-buckling phases. This indicates that when buckling initiates the deformation 


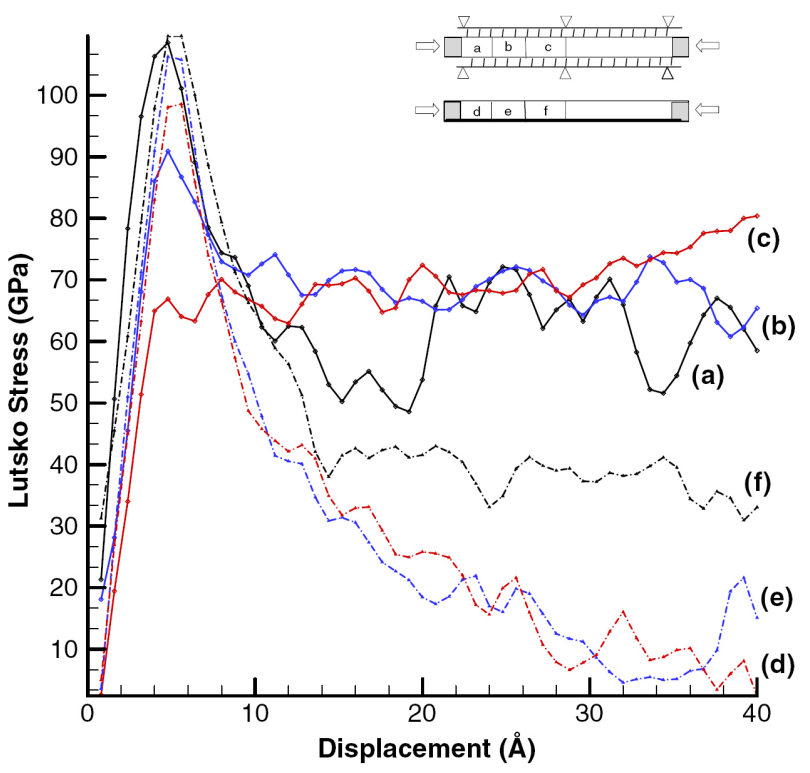

Figure 7. Lutsko stress of various regions for CNT with strong interface $(\mathrm{a}-\mathrm{c})$ and functionalized CNT $(\mathrm{d}-\mathrm{f})$. The inset shows schematically the regions to which each plot corresponds.

occurs mainly near the loading ends of nanotube. This deformation results in extension of bonds and load transfer to the butene chemical attachments in this region. Once the chemical attachments transfer the load to the matrix, the deformation of nanotube is fairly localized; the central region then is not loaded. Figure 8 shows simulation snapshots of nanotubes for different boundary conditions. It can be observed that the deformation in neat nanotubes and functionalized nanotubes with matrix end free is similar.
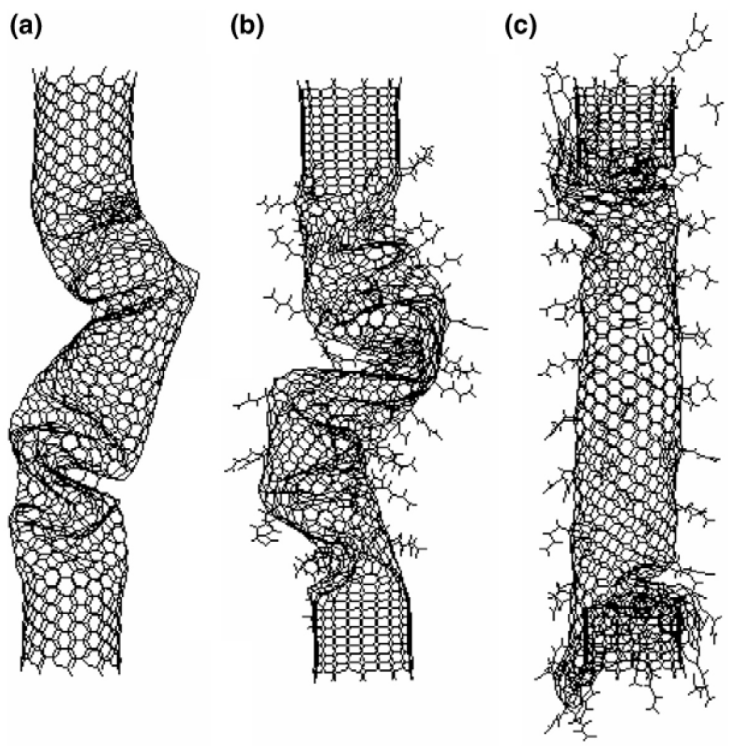

Figure 8. Simulation snapshots of $(10,10)$ CNT subject to compression (a) is the free CNT, (b) is functionalized CNT (c) CNT with chemically bonded interface. Notice the change in deformation mechanism to crushing in (c).
However, in the case of nanotube with matrix end of chemical attachment fixed, extensive deformation resulting in crushing occurs at ends while the central region is relatively undisturbed. Because of the nature of the chemically bonded interface, the portion of nanotube near the ends act as fiber with very small aspect ratio and experience crushing under compressive load.

In order to examine the validity of boundary condition for more general conditions and to study the effect of the length of hydrocarbon chemical attachments, the above simulations for 80 hydrocarbon chains are performed with octene and hexene chemical attachments. Figure 9 shows the compressive stress vs. displacement plots for butene, hexene and octene chemical attachments. In all the cases, there is a decrease in the critical stress for buckling followed by a crushing type of post-buckling deformation. This shows that this is the general behavior of composite with chemically bonded interface.

An interesting phenomenon is observed in atomic scale interfaces which do not occur in continuous interfaces. The drop and rise in stress marked as region 1 in Figure 5 is because of the failure of individual chemical attachments in the interface region. Though general dynamical behavior will always show a fluctuating behavior, this sharp rise is clearly different. We observe that chemical attachments do not fail suddenly; instead they often experience debonding and rebonding with adjacent atoms before failure. Figure 10 shows the reaction force experienced by the fixed atoms along the length of the nanotube averaged over every 100 time steps. The reaction force increase for individual attachments occurs discontinuously with peaks and troughs instead of a monotonous increase. This is because the chemical attachments which debond from the carbon nanotube re-attach with adjoining atom of nanotube (as shown in the inset of Figure 10) before they fail completely. This behavior is more prominent in tensile loading [32] but seems to occur in compressive loading as well.

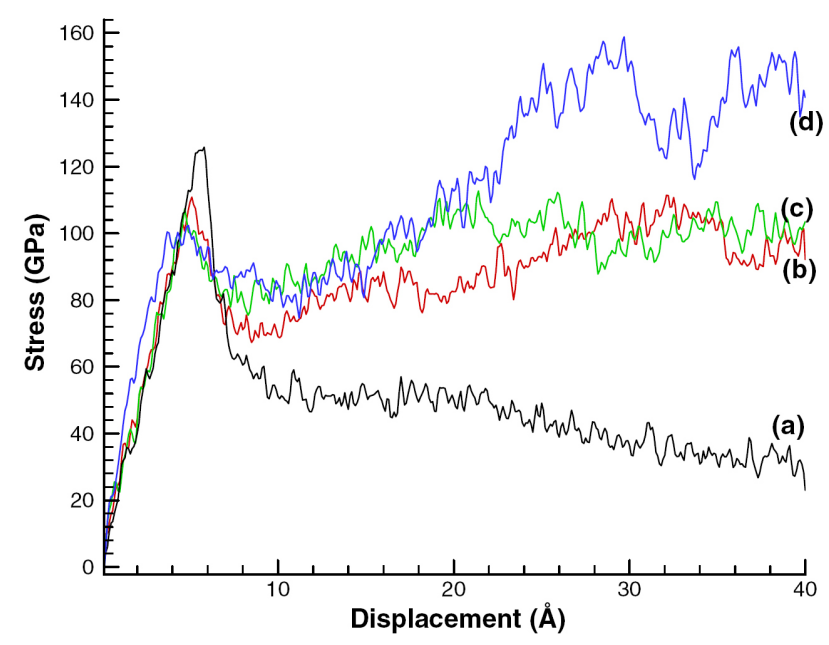

Figure 9. Compressive stress vs. displacement for CNT with 80 hydrocarbon chemical attachments of different lengths (a) neat CNT, (b) octene attachments, (c) hexene attachments and (d) butene attachments. All the attachments are fixed at ends. 


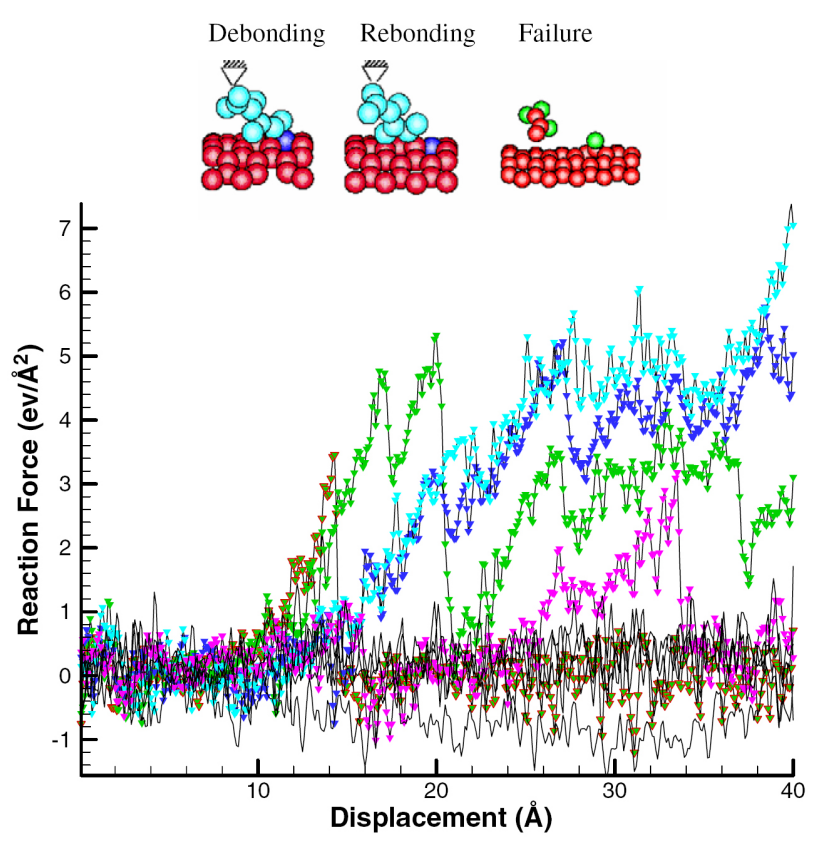

Figure 10. Reaction force experienced by fixed atoms along the length of CNT. Inset shows a schematic of debonding and rebonding.

These observations indicate that the interfacial behavior at nanoscale is different from that of conventional composites. Compared to continuous interfaces where the load transfer occurs instantly due to the presumed material continuity, the mechanism of load transfer is different in discrete atomic systems. Here, the independent bonds rotate and align until the bonds are stretched. From geometric conditions nanotubes are sufficiently deformed only during the post-buckling stage of loading. The stretching of the bonds in chemical attachments and hence load transfer depends on several factors such as density of chemical attachments, distance between fiber and matrix, length and type of chemical attachments, etc. In addition, factors such as debonding and rebonding of chemical attachments affect the mechanism load transfer in nanoscale interfaces.

\section{Summary}

Buckling and post-buckling behavior of carbon nanotube fiber embedded in matrix has been investigated for non-bonded and bonded interfaces. Summarizing:

- There is only a small difference in critical stress for neat nanotubes and nanotubes embedded in polythene matrix. This difference is much less than that predicted by continuum equations.

- In the case of chemically bonded interfaces the critical stress for buckling is reduced compared to neat nanotubes because of the changes in curvature introduced by chemical bonding

- In the post-buckling phase chemically bonded nanotubes act as short fibers and deform by crushing.
Acknowledgment

Financial support from ARO (Dr. Bruce La Mattina) and AFOSR (Dr. Les Lee) is gratefully acknowledged.

\section{References}

[1] M.M.J. Treacy, T.W. Ebbesen, and J.M. Gibbson, Exceptionally high Young's modulus observed for individual carbon nanotubes, Nature 381 (1996), p. 678.

[2] D.H. Robertson, D.W. Brenner, and J.W. Mintmire, Energetics of nanoscale graphitic tubules, Phys Rev B 45 (1992), p. 12592.

[3] Engineered materials handbook, vol. 1, Composites. Metals Park, Ohio: ASM International; 1987.

[4] B.G. Demczyk, Y.M. Wang, J. Cumings, M. Hetman, W. Han, and A. Zettl et al., Direct mechanical measurement of the tensile strength and elastic modulus of multiwalled carbon nanotubes, Mater Sci Eng A 334 (2002), p. 173. ics of carbon tubes: instabilities beyond linear response, Phys Rev Lett 76 (1996), p. 2511.

[6] T. Belytschko, S.P. Xiao, G.C. Schatz, and R.S. Ruoff, Atomistic simulations of nanotube fracture, Phys Rev B 65 (2002), p. 235430.

[7] Q.H. Wang, A.A. Setlur, J.M. Lauerhaas, J.Y. Dai, E.W. Seelig, and R.P.H. Chang, A nanotube-based field-emission flat panel display, Appl Phys Lett 72 (1998), p. 2912.

[8] Z.W. Pan, S.S. Xie, L. Lu, B.H. Chang, L.F. Sun, and W.Y. Zhou et al., Tensile tests of ropes of very long aligned multiwalled carbon nanotubes, Appl Phys Lett 74 (1999), p. 3152.

[9] D. Qian, E.C. Dickey, R. Andrews, and T. Rantell, Load transfer and deformation mechanisms in carbon nanotubepolystyrene composites, Appl Phys Lett 76 (2000), p. 2868.

[10] E.W. Wong, P.E. Sheehan, and C.M. Lieber, Nanobeam mechanics: elasticity, strength, and toughness of nanorods and nanotubes, Science 277 (1997), pp. 1971-1975.

[11] M.B. Nardelli, B.I. Yakobson, and J. Bernhloc, Mechanism of strain release in carbon nanotubes, Phys Rev B 57 (1998), pp. R4277-R4280.

[12] H.J. Dai, J.H. Hafner, A.G. Rinzler, D.T. Colbert, and R.E. Smalley, Nanotubes as nanoprobes in scanning probe microscopy, Nature 384 (1996), pp. 147-150.

[13] S. Iijima, C. Brabec, A. Maiti, and J. Bernholc, Structural flexibility of carbon nanotubes, J Chem Phys 104 (1996), pp. 2089-2092.

[14] L.S. Schadler, S.C. Giannaris, and P.M. Ajayan, Load transfer in carbon nanotube epoxy composites, Appl Phys Lett 73 (1998), p. 3842.

[15] O. Lourie, D.M. Cox, and H.D. Wagner, Buckling and collapse of embedded carbon nanotubes, Phys Rev Lett 81 (1998), p. 1638.

[16] V.M. Harik, Mechanics of carbon nanotubes: applicability of the continuum-beam models, Comput Mater Sci 24 (2002), p. 328.

[17] C.Q. Ru, Effect of van der Waals forces on axial buckling of a double-walled carbon nanotube, J Appl Phys 87 (2000), p. 7227. 
[18] C.Q. Ru, Axially compressed buckling of a double-walled carbon nanotube embedded in an elastic medium, J Mech Phys Solids 49 (2001), p. 1265.

[19] C. Li and T.W. Chou, Modeling of elastic buckling of carbon nanotubes by molecular structural mechanics approach, Mech Mater 36 (2004), p. 1047.

[20] A. Garg and S.B. Sinnott, Effect of chemical functionalization on mechanical properties of carbon nanotubes, Chem Phys Lett 295 (1998), p. 273.

[21] S. Govindjee and J.L. Sackman, On the use of continuum mechanics to estimate the properties of nanotubes, Solid State Commun 110 (1999), p. 227.

[22] L.J. Sudak, Column buckling of multiwalled carbon nanotubes using nonlocal continuum mechanics, J Appl Phys 94 (2003), p. 7281.

[23] Z. Jia, Z. Wang, C. Xu, J. Liang, B. Wei, and D. Wu et al., Study on poly(methyl methacrylate) carbon nanotube composites, Mater Sci Eng A 271 (1999), p. 395.

[24] C.A. Cooper, R.J. Young, and M. Halsall, Investigation into the deformation of carbon nanotubes and their composites through the use of Raman spectroscopy, Composites: Part A 32 (2001), p. 401.

[25] W. Ding, A. Eitan, F.T. Fisher, X. Chen, D.A. Dikin, and R. Andrews et al., Direct observation of polymer sheathing in carbon nanotube-polycarbonate composites, Nano Lett 3 (2003), p. 1593.

[26] P.M. Ajayan, L.S. Schadler, C. Giannaris, and A. Rubio, Single-walled carbon nanotube-polymer composites: strength and weakness, Adv Mater 12 (2000), p. 750.

[27] R. Andrews, D. Jacques, M. Minot, and T. Rantell, Fabrication of carbon multiwalled nanotube/polymer composites by shear mixing, Macromol Mater Eng 287 (2002), p. 395.

[28] E.T. Michelson, C.B. Huffman, A.G. Rinzler, R.E. Smalley, R.H. Hauge, and J.L. Margrave, Fluorination of single-wall carbon nanotubes, Chem Phys Lett 296 (1998), p. 188.

[29] S. Pekker, J.P. Salvetat, E. Jakab, J.M. Bonard, and L. Forro, Hydrogenation of carbon nanotubes and graphite in liquid ammonia, J Phys Chem B 105 (2001), p. 7938.

[30] J. Chen, M.A. Hamon, H. Hu, Y. Chen, A.M. Rao, and P.C. Eklund et al., Solution properties of single-walled carbon nanotubes, Science 282 (1998), p. 95.
[31] A. Eitan, K. Jiang, D. Dukes, R. Andrews, and L.S. Schadler, Surface modification of multiwalled carbon nanotubes: toward the tailoring of the interface in polymer composites, Chem Mater 15 (2003), p. 3198.

[32] S. Namilae and N. Chandra, Multiscale model to study the effect of interfaces in carbon nanotube based composites, ASME J Eng Mater Technol 127 (2005), pp. 222-232.

[33] D.W. Brenner, Empirical potential for hydrocarbon for use in simulating the chemical vapor deposition of diamond films, Phys Rev B 42 (1991), p. 9458.

[34] D.W. Brenner, O.A. Shenderova, J.A. Harrison, S.J. Stuart, B. Ni, and S.B. Sinnott, A second-generation reactive empirical bond order (REBO) potential energy expression for hydrocarbons, J Phys-Condens Matter 14 (2002), pp. 783-802.

[35] N. Chandra, S. Namilae, and C. Shet, Local elastic properties of carbon nanotubes in the presence of Stone-Wales defects, Phys Rev B 69 (2004), p. 094101.

[36] E.R. Williams, G.C. Jones Jr., L. Fang, R.N. Zare, B.J. Garrison, and D.W. Brenner, Ion pickup of large, surface-adsorbed molecules - a demonstration of the Eley-Rideal mechanism, J Am Chem Soc 114 (1992), p. 3207.

[37] L.A. Girifalco, M. Hodak, and R. Lee, Carbon nanotubes, buckyballs, ropes, and a universal graphitic potential, Phys Rev B 62 (2000), p. 13104.

[38] Bulson PS. Buried structures; 1985.

[39] A.C. Ugural, and S.K. Fenster, Advanced strength and applied elasticity, Prentice Hall, New Jersey (2003).

[40] Y. Lanir and Y.C.B. Fung, Fiber composite columns under compression, J Compos Mater 6 (1972), p. 387.

[41] N. Chandra and H. Ghonem, Interfacial mechanics of push-out tests: theory and experiments, Composites: Part A 32 (2001), p. 575.

[42] J.F. Lutsko, Stress and elastic-constants in anisotropic solids - molecular dynamics, J Appl Phys 64 (1998), p. 1152.

[43] S. Namilae, N. Chandra, and C. Shet, Mechanical behavior of functionalized nanotubes, Chem Phys Lett 387 (2004), p. 247. 